\title{
The reduction in anemia through normative innovations (RANI) project: study protocol for a cluster randomized controlled trial in Odisha, India
}

\author{
Hagere Yilma ${ }^{1 *} \mathbb{D}$, Erica Sedlander ${ }^{1}$, Rajiv N. Rimal ${ }^{2}$, chhya Pant $^{1}$, Ashita Munjral ${ }^{3}$ and Satyanarayan Mohanty ${ }^{4}$
}

\begin{abstract}
Background: More than half of women in India are anemic. Anemia can result in fatigue, poor work productivity, higher risk of pre-term delivery, and maternal mortality. The Indian government has promoted the use of iron-folic acid supplements (IFA) for the prevention and treatment of anemia for the past five decades, but uptake remains low and anemia prevalence high. Current programs target individual-level barriers among pregnant women and adolescents, but a more comprehensive approach that targets multiple levels among all women of reproductive age is needed to increase uptake of IFA and iron-rich foods.

Methods: The Reduction in Anemia through Normative Innovations (RANI) project is a norms-based intervention to reduce anemia among women of reproductive age. We will evaluate the intervention through a clustered randomized controlled trial in Odisha, India. We will collect data at three time points (baseline, midline, and end line). For the study, we selected 89 clusters of villages, which we randomized into treatment and control on a 1:1 basis. The treatment arm will receive the RANI project components while the control arm will receive usual care. Fifteen clusters (40-41 villages) were selected and 4000 women (2000 in each arm) living in the selected clusters will be randomly selected to take part in data collection. Women in both study arms will have their hemoglobin concentrations measured. They will also complete in-person surveys about their knowledge, attitudes, perceptions of iron folic acid supplements, and nutritional intake. We will also select a smaller cohort of 300 non-pregnant women (150 in each arm) from this cohort for additional physical activity and cognitive testing. We will conduct both within- and between-group comparisons (treatment and control) at baseline, midline and end line using t-tests. We will also conduct structural equation modeling to examine how much each factor accounts for IFA use and hemoglobin levels.
\end{abstract}

Discussion: This RCT will enable us to examine whether a social norms-based intervention can increase uptake of iron folic acid supplements and iron rich foods to reduce anemia.

Trial registration: This trial was registered with Clinical Trial Registry- India (CTRI) (CTRI/2018/10/016186) on 29 October 2018.

Keywords: Social norms, Iron deficiency anemia, Cluster randomization, Controlled trial

\footnotetext{
* Correspondence: hyilma@gwu.edu

${ }^{1}$ Department of Prevention and Community Health, George Washington University Milken Institute School of Public Health, Washington, District of Columbia, USA

Full list of author information is available at the end of the article
}

(c) The Author(s). 2020 Open Access This article is distributed under the terms of the Creative Commons Attribution 4.0 International License (http://creativecommons.org/licenses/by/4.0/), which permits unrestricted use, distribution, and reproduction in any medium, provided you give appropriate credit to the original author(s) and the source, provide a link to the Creative Commons license, and indicate if changes were made. The Creative Commons Public Domain Dedication waiver (http://creativecommons.org/publicdomain/zero/1.0/) applies to the data made available in this article, unless otherwise stated. 


\section{Background}

Anemia is a serious health concern in India, where more than half of women of reproductive age (WRA) are anemic [1]. It is mostly associated with fatigue and thus poor work productivity [2], but if left untreated, anemia can lead to poor birth outcomes, including higher risk for preterm delivery and maternal mortality [3]. Anemia during pregnancy can also inhibit physical and cognitive development in children [4-6].

In Odisha, India (the site of this study) the majority of anemia cases are a result of iron-deficiency, due to poor dietary iron intake, low iron absorption, and iron-loss during intestinal worm infection, pregnancy and menstruation. As one of six Global Nutrition Targets for 2025, the World Health Organization (WHO) has set forth a series of recommendations to prevent and reduce anemia [7]. Among these recommendations is regular iron-folic acid (IFA) supplementation for all women of reproductive age between 15 and 40 years old (including pregnant and nonpregnant women) in regions where more than $40 \%$ of women are anemic [7].

India has implemented several national-level programs to increase IFA supply over the last 50 years. However, anemia levels remain high, partly because of the scarcity of interventions to improve the demand for and uptake of IFA and iron-rich foods. Of late, efforts to promote IFA consumption in India have adopted a life cycle approach by including women of reproductive age (nonpregnant and non-lactating) for IFA supplementation rather than exclusively targeting pregnant and lactating women, adolescents and/or children [8,9]. Unlike pregnant women, non-pregnant and out-of-school women are poorly served as the government is currently in the process of rolling out its IFA supplementation strategy to these important sub-populations. Not surprisingly, adherence rates in this group is also poorly understood. For example, the Indian National Family Health Survey (NFHS) collects data on IFA adherence only for pregnant women [1]. Nevertheless, available data indicate that adherence is poor as only $30.3 \%$ of mothers in India reported consuming IFA for 100 days or more when they were pregnant, although 91\% percent received IFA [1]. To effectively reduce anemia in India, both pregnant and non-pregnant WRA should not only receive IFA, but they should also take it regularly.

Innovative approaches that increase IFA demand can propel changes at multiple levels (individual, interpersonal, community and policy). Shet et al. [10] demonstrated that educational counseling delivered to mothers and caregivers can improve IFA consumption and reduce anemia in children. Behavior change interventions that target the individual directly are also effective in improving IFA consumption. Adolescent girls in Delhi showed improvement in their IFA consumption, along with their knowledge and attitudes around IFA and anemia, after receiving an educational intervention delivered in schools [11]. Many IFA-focused interventions in India that target adolescent school girls have also been successful in reducing anemia prevalence through supplementing IFA provision with educational information [12]. While programs of this sort for adult women are limited, a similar communication intervention delivered through women's Self Help Groups to pregnant women in rural India was effective in improving IFA consumption among other pregnancy-related behaviors [13]. While the vast majority of behavior change interventions that promote IFA consumption target in-school girls or pregnant women, they should be extended and adapted for all WRA, regardless of pregnancy or school status.

The Reduction in Anemia through Normative Innovations (RANI) Project aims to reduce the burden of anemia among all WRA in India through a social norms-based approach. Social norms are based on the idea that people conform to the behaviors they perceive others around them are engaging in. Thus, the extent to which WRA believe others are taking IFA can influence their own IFA consumption. The theoretical underpinnings of the intervention are described in greater detail in later sections. In this paper, we describe the randomized control trial protocol, which we are using to test the efficacy of the RANI Project in increasing IFA and iron rich food consumption to reduce anemia among WRA.

\section{Objectives}

The objective of this study is to investigate the ability of a norms-based behavior change intervention to reduce anemia among women of reproductive age in Odisha, India. We will test the following hypotheses:

$\mathrm{H} 1$. Changes in women from baseline to end line in the intervention arm will be significantly greater than corresponding changes in the control arm in the following outcomes: (a) anemia status, (b) IFA use, (c) mental health/ depression, (d) physical activity (e) work capacity, (f) consumption of iron rich foods and ( $\mathrm{g}$ ) cognitive functioning;

$\mathrm{H} 2$. Social norms serve as a mediator in the relationship between intervention exposure and study outcomes; and.

H3. Changes in women baseline to end line in the intervention arm will be significantly greater than corresponding changes in the control arm in knowledge, attitudes, perceptions, consumption of iron-rich foods, and use of IFA.

\section{Methods}

\section{Study setting}

We will conduct the study in Odisha, which is on the eastern coast of India, where $83 \%$ of residents live in rural areas. Across Odisha, 94\% of households are Hindu and $23 \%$ belong to a tribal culture. Around 
three fourths $(73 \%)$ of the total population and nearly two-thirds (64\%) of women in Odisha are literate [14]. Additionally, the total fertility rate (TFR) is approximately 2.1 children per woman in Odisha. Around half of WRA in Odisha are anemic (51.0\%). The prevalence of anemia among women is high across subgroups: those who are breastfeeding (54.8\%), pregnant $(47.6 \%)$, and WRA who are neither breastfeeding nor pregnant (50.3\%). Women with less education and who belong to Scheduled Tribes are more likely to be anemic [1].

Within Odisha, we chose Angul district for our study site because its anemia prevalence is similar to that of the state and the rest of India [1]. Anemia rates and IFA adherence in Angul also follow a similar pattern to Odisha State: $44.0 \%$ of the Angul population is anemic and only $38 \%$ of women consume IFA when they were pregnant. We selected two blocks within the Angul district, Athmallik and Kishorenagar, as study sites (a block is the administrative unit larger than a village but smaller than a district and each block encompasses several villages). The two blocks were not randomly selected, rather they were selected because they are adjacent to each other, spread over an area of 1278 sq. kilometers (499 sq. miles) and are representative of the district. According to the 2011 census, the two blocks have a total of 588 villages, accounting for a total of 218,373 people in 50,463 households [14]. In the two blocks, nearly one fourth of people are tribal, a third are literate, and about half of women work outside of the home.

\section{Design}

We will use a cluster randomized controlled trial (RCT) design. In this design, villages will be randomized on a 1:1 ratio to receive the treatment or continue with usual care (defined as the currently existing and ongoing efforts to reduce anemia in Odisha). Treatment is defined as exposure to one or more components of the RANI project. As this is a community-level intervention, we used a cluster design to prevent contamination across communities.

We grouped together villages into clusters of 1-4 villages, resulting in eighty-nine total clusters. A geographical buffer of at least one village or natural structure (e.g., a mountain) was maintained between clusters to limit contamination. We first used a random number generator to randomly assign clusters into treatment ( $k=50$ clusters $)$ and control arms ( $k=$ 38 clusters). Clusters were randomly given a value of ' 1 ' or ' 2 '; those that were given a ' 1 ' were assigned to the treatment and those that were given a ' 2 ' were assigned to the control. Thus, the number of clusters in each arm are not exactly equal.
We then segmented clusters by proportion of minority populations (in India, they are called scheduled castes and scheduled tribes) and then selected three clusters from each stratum for data collection so that 15 clusters (which comprised 41 villages) from the treatment arm and 15 (comprising 40 villages) from the control arm were selected for data collection. The decision to select a smaller subset of 15 clusters from each arm for data collection was made in order to maximize the sample size per cluster. Data collectors and program implementers will be blinded with regard to treatment and control status of villages. Data collection will occur at three time points: baseline, midline, and end line. The overall schema of the study design is depicted in Fig. 1.

\section{Participants}

All members of the treatment clusters meeting the inclusion criteria will be eligible to participate in the trial.

\section{Inclusion criteria}

All women selected for data collection must be between 15 and 49 years old, a resident of the village, and speak Odiya. Additionally, as this is a longitudinal study, participants must indicate that they are not planning to move out of the village for the next two years.

\section{Exclusion criteria}

We will exclude women with an active fever at data collection and refer them to the closest health center, as the interview may take up to an hour or longer and may exacerbate any illness they may already have. Once excluded from baseline, the woman will no longer be eligible for data collection and midline and endline. However, women who are excluded from data collection may still be reached in the intervention if they live in a treatment village. We will also refer those with severe anemia to the local health center, but they will not be excluded from data collection. Though pregnancy status is not an inclusion or exclusion criterion, we will exclude currently pregnant women from certain components of data collection that could put them at risk - they will only take part in the survey and provide hemoglobin measurements.

\section{Rational and overview of the intervention}

We developed the intervention based on the literature as well as findings from our formative research to understand barriers to and facilitators of IFA use. The formative research [15] comprised the following components:

- 16 focus group discussions with women of reproductive age, their husbands, and mothers-inlaw 


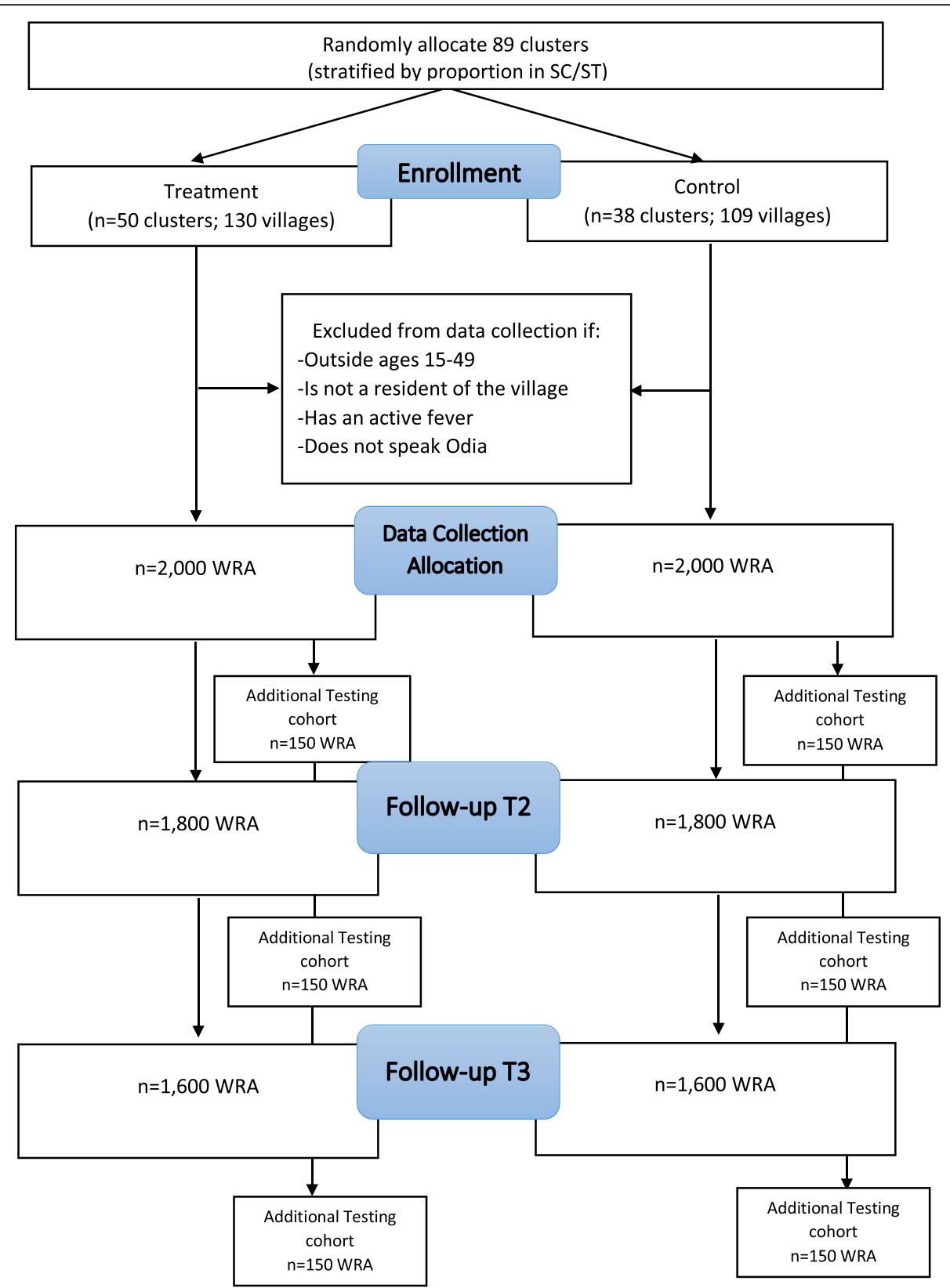

Fig. 1 Custer Randomized Control Trial Schema

- 25 key informant interviews with self-help group leaders, medical doctors, teachers, natural healers, and frontline health workers

- A perceptual mapping exercise to understand how women of reproductive age, their mothers-in-law, and their husbands conceptualize IFA and other anemia-related items (e.g., fatigue, fruits and vegetables, medical care, etc.)

\section{Formative research results}

The formative research provided insights at multiple levels. At the individual level, we learned that the majority of people had basic knowledge about anemia and knew that IFA can prevent and treat anemia. However, women did not have a clear understanding about their own anemia risk; rather, they had normalized the existence of milder forms of anemia. We also identified both real and perceived side effects of IFA use, including some misperceptions.

At the interpersonal level, we found that perceptions of approval from referent groups (i.e., husbands and mothers-in-law) played a major role in women's decisions to take IFA. These referent groups, largely mothers-inlaw, were also found to perpetuate misconceptions around 
IFA use, including the belief that taking IFA during pregnancy would result in an abnormally large baby during and thus complicating delivery.

At the community level, we found that women's health was not a priority and women were expected to take care of their families before thinking about their own well-being. They were also expected to work for the household all day, leaving little time for themselves, thus reducing their ability to visit a health center to get tested for anemia or to obtain IFA.

At the policy level, we found that out-of-school non-pregnant women were not directly served by existing government practices of delivering IFA. Health workers distribute IFA to pregnant women in their homes and in village health and nutrition days, and adolescents obtain them in schools; non-pregnant women do not know that they should be taking IFA weekly (per Indian government guidelines) and therefore do not seek it out $[8,9,16]$.

\section{The role of social norms}

The proposed intervention will use a social norms approach to incorporate factors at play at multiple levels of the socio-ecological model. Social norms are based on the idea that people change behaviors because they perceive that others around them are changing and they do not want to be left behind. Descriptive norms refer to people's perceptions about the prevalence of a behavior - what they believe others are doing - and injunctive norms refer to pressures people feel to conform [17]. Additionally, collective norms refers to the actual prevalence of behavior among one's peers (e.g., the actual number of women taking IFA in a woman's village) [18].

\section{Theoretical underpinning}

The RANI project intervention is based on the theory of normative social behavior (TNSB), which posits that social norms drive behavior and that this influence is further heightened when moderators are in favor of the behavior [19]. According to the TNSB, the relationship between social norms and behavior is moderated by a number of factors, including behavioral (e.g., access and outcome expectations), individual-level (e.g., self-efficacy, knowledge, and risk perception), and contextual-level (e.g., interpersonal discussion, gender norms, and nutrition) factors. Following the theoretical guidelines, this project will focus on descriptive norms (perceived prevalence), injunctive norms (pressures people feel to conform), and collective norms (actual prevalence) surrounding IFA consumption.

The TNSB also posits that norms, by themselves, may not be enough to propel change [20]; normative information often must be coupled with information about benefits of performing the behavior [21], the behavior itself must be easy to enact [22], and people must be convinced that others in their social network are also engaging in the behavior [23]. Thus, if people learn that others in their social network are taking IFA, that they themselves can also take them, and that these supplements have benefits (e.g., improving their health or providing them with more energy), they may be persuaded to do the same. The overall theory of change for the intervention can be found in Fig. 2. The consideration of the potential moderators that can propel norms into action can help combat attributable barriers of IFA consumption, such as unpleasant side effects. For example, we know from our formative research that women prioritize their ability to help their family. Guided by TNSB, we suspect that when positive descriptive norms around IFA (i.e., the belief that other WRA are taking IFA) are coupled with positive injunctive norms (i.e., perceptions of support from their mother-in-laws and husbands) and strong risk perceptions and other psychosocial factors related to anemia and IFA, norms may translate into IFA consumption despite the barriers related to side effects.

Our approach will focus on generating demand at multiple levels. At the individual level, we will raise awareness and knowledge around anemia, correct misperceptions about the role of iron (in making deliveries more difficult), increase risk perception (susceptibility and severity), and enhance self-efficacy. At the interpersonal level, we will promote positive social norms around taking IFA and eating iron rich foods, along with other foods that promote iron absorption. We will focus on improving descriptive norms through demonstration events in communities in which women take IFA in a public setting and where community-level hemoglobin counts are graphically displayed. To improve injunctive norms, we will focus on persuading women's husbands and their mothers-in-law to support them taking IFA. At the policy level, we will engage with health officials at multiple levels and policymakers at the state level to ensure that they are promoting IFA guidelines, that there is a continuous supply of IFA, and that they are promoting demand-generation efforts. A description of all RANI activites can be found in Table 1, along with the timeline for the intervention (Table 2).

To catalyze individual-level change, the intervention will use a T4 approach: Train, Tell, Test, and Tweak. We will train WRA and other community members through self-help group (SHG) meetings about anemia, IFA, and iron-rich foods so they can bring this knowledge to their community. SHGs are the primary platform of women's empowerment across India. Within each village, several SHGs convene regularly to empower women with financial literacy and other forms of 


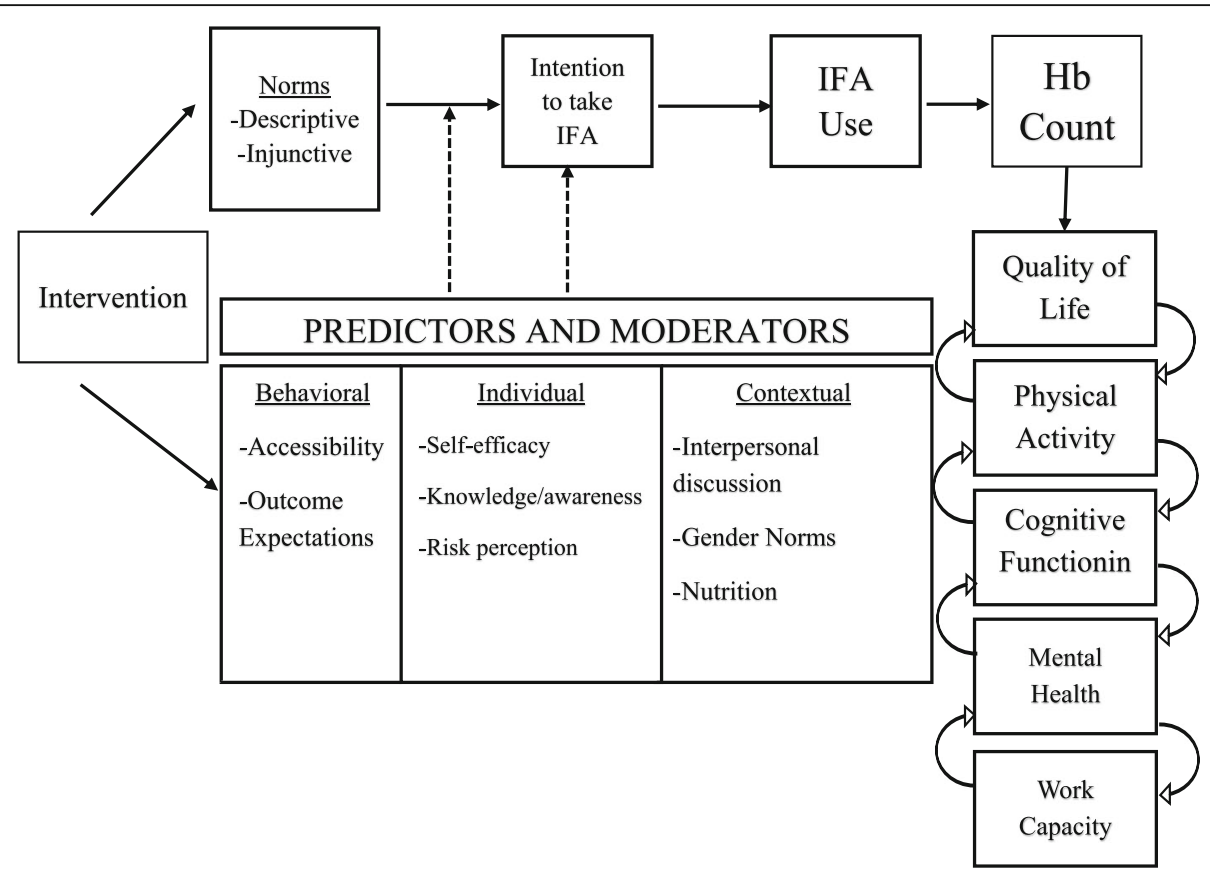

Fig. 2 Theory of Change

support. The involvement of SHGs in the intervention comes through our partnership with the Odisha Livelihood Mission (OLM), the government organization responsible for the formation and management of women SHGs in the state. We will develop ten modules that will include a mix of didactic learning and games focused on specific behavior changes and then use the SHG platform to conduct follow-up sessions in small groups.

The intervention will also tell the stories of overcoming barriers to IFA use through six short videos that feature members of the target audiences (WRA, husbands, mothers-in-law, and frontline workers) overcoming the barriers that we identified in the formative research. We will show the videos during SHG meetings, village health and nutrition days, and community festivals. The goal of the videos is to promote collective interest around anemia prevention by increasing knowledge, improving risk perception, enhancing perceptions of efficacy, and promoting positive social norms. We will also send regular voice-based messages to mobile phones to remind women to take the IFA, and we will also reinforce social norms around taking them.

We plan to test WRA both in the SHGs and throughout the community for anemia via a point-of-care hemoglobin test. We will then display the individual- and SHG- level results in the community, using graphic methods appropriate for low-literacy audiences.. The goal of this activity is to promote three types of feedback - ipsative (comparisons between community hemoglobin levels in the past and the present), social (how two neighboring communities are faring, compared to participants' own community), and aspirational (how the community is faring, compared to goals set by the community early on).

Based on continuous monitoring and evaluation, we will tweak the curriculum, messages, and/or overall approach. We will gather real-time quantitative and periodic qualitative data about each intervention component to ensure fidelity and to gather feedback about which areas are working and which areas need improvement. The qualitative data will also capture unintended consequences (both positive and negative) as a result of the intervention.

\section{Outcomes}

The primary evaluation outcome is anemia among women of reproductive age, defined as having hemoglobin count less than $12 \mathrm{~g} / \mathrm{dcl}$ among non-pregnant women and less than 11 $\mathrm{g} / \mathrm{dcl}$ among pregnant women. We will measure this via a HemoCue point-of-care blood prick. We will also measure self-reported IFA use via a tablet-based survey.

Several secondary outcomes will also be measured in all participants to understand the mechanism of change, including: (a) knowledge and perceptions about anemia and IFA, (b) social norms, (c) diet, (d) mental health, and (e) quality of life. In a smaller sample of non-pregnant women, we will assess other secondary outcomes, including (a) physical activity (through ActivPal readings), (b) work capacity (through the modified Queens College step test), and (c) socio-cognitive functioning (through paper and computer-based response time tasks). 
Table 1 RANI Intervention Activities

\begin{tabular}{|c|c|c|c|c|}
\hline $\begin{array}{l}\text { Intervention } \\
\text { Component }\end{array}$ & Description & Objectives & Dose & Delivered by \\
\hline $\begin{array}{l}\text { T4 Sessions and } \\
\text { Community } \\
\text { Engagement } \\
\text { Meetings }\end{array}$ & $\begin{array}{l}10 \text { Participatory Learning and Action (PLA) } \\
\text { sessions and community engagement } \\
\text { meetings developed in collaboration with our } \\
\text { implementation partner and another local } \\
\text { partner specializing in the methodology based } \\
\text { in Delhi. } \\
\text { Sessions and community engagement } \\
\text { meetings include a mix of didactic learning } \\
\text { and games focused on specific topics related } \\
\text { to anemia prevention and theoretical } \\
\text { constructs. } \\
\text { Target population: women of reproductive } \\
\text { age (WRAs), mothers-in-law, husbands, front- } \\
\text { line workers, and government officials/policy } \\
\text { makers }\end{array}$ & $\begin{array}{l}\text { The sessions will cover information related to } \\
\text { anemia, knowledge and awareness about iron } \\
\text { folic acid (IFA) supplements, dietary diversity, } \\
\text { social norms, malaria, water and sanitary } \\
\text { hygiene (WASH), and deworming. }\end{array}$ & Monthly & $\begin{array}{l}\text { RANI } \\
\text { Community } \\
\text { Facilitators }\end{array}$ \\
\hline Rani Comm & $\begin{array}{l}6 \text { media products - 3-4 min locally shot videos } \\
\text { complimenting the concept developed in col- } \\
\text { laboration with our implementation partners } \\
\text { and a local media production house based in } \\
\text { Delhi. } \\
\text { We show videos to small groups on smart } \\
\text { phones. } \\
\text { In year three, we will have evening viewings } \\
\text { on large projectors in each village. } \\
\text { Target population: women of reproductive } \\
\text { age (WRAs), mothers-in-law, and husbands }\end{array}$ & $\begin{array}{l}\text { Each video highlights the key messages of the } \\
\text { program (including modeling positive social } \\
\text { norms around IFA) and addresses the myths } \\
\text { and barriers that we identified during the } \\
\text { formative research around anemia/IFA } \\
\text { consumption. }\end{array}$ & Ongoing basis & $\begin{array}{l}\text { RANI } \\
\text { Community } \\
\text { Facilitators }\end{array}$ \\
\hline $\begin{array}{l}\text { Hemoglobin } \\
\text { Testing and } \\
\text { Demonstration }\end{array}$ & $\begin{array}{l}\text { Fifteen women from each of the } 130 \\
\text { intervention villages will undergo monthly } \\
\text { hemoglobin testing in their village ( } n=1950 \\
\text { women per month). The testing is user- } \\
\text { friendly with instant digital results. } \\
\text { Their Hemoglobin levels along with their IFA } \\
\text { consumption status will also be tracked on a } \\
\text { monthly basis. We designed cards with } \\
\text { different colors indicating anemia severity, } \\
\text { (green, yellow, orange, red), along with } \\
\text { relevant behavioral nudges to share the } \\
\text { Hemocue results. We use individual tracking } \\
\text { cards to monitor hemoglobin progress. We } \\
\text { share results at the individual, group, and } \\
\text { inter-village level to trigger demand for IFA } \\
\text { uptake and consumption of iron rich foods. } \\
\text { Target population: women of reproductive } \\
\text { age (WRAs) for testing and their families/ } \\
\text { villages for demonstrations }\end{array}$ & $\begin{array}{l}\text { The goal of this activity is to promote three } \\
\text { types of feedback - ipsative (comparisons } \\
\text { between community hemoglobin levels in the } \\
\text { past and the present), social (how two } \\
\text { neighboring communities are faring, } \\
\text { compared to participants' results at the } \\
\text { individual, group, and inter-village level to trig- } \\
\text { ger demand for IFA uptake and consumption } \\
\text { of iron rich foods. }\end{array}$ & Monthly & $\begin{array}{l}\text { RANI } \\
\text { Community } \\
\text { Facilitators }\end{array}$ \\
\hline mRANI & $\begin{array}{l}\text { mRANI or mobile-RANI is a smaller interven- } \\
\text { tion built into the larger RANI trial to increase } \\
\text { demand and adherence to IFA supplements } \\
\text { using interactive norms-based audio } \\
\text { messages. } \\
\text { After midline data collection (Spring 2020), we } \\
\text { will begin the 12-week intervention. We will } \\
\text { send audio messages via automated phone } \\
\text { calls. As this is an interactive dialogue, we will } \\
\text { encourage participants to ask questions, seek } \\
\text { additional information, share their experience, } \\
\text { or provide feedback via text or phone call. We } \\
\text { will use an open-source two-way Interactive } \\
\text { Voice Response (IVR) system. We chose audio } \\
\text { recordings to be able to reach women with } \\
\text { low literacy in an approachable, efficient, and } \\
\text { cost-effective way. } \\
\text { Target population: women of reproductive } \\
\text { age (WRAs) }\end{array}$ & $\begin{array}{l}\text { The primary objective of mRANI is to examine } \\
\text { the effectiveness of automated voice call } \\
\text { messages with a social-norms framing to in- } \\
\text { crease IFA demand among women living in } \\
\text { low-resource settings. } \\
\text { Each call will be } 30 \text { s long and enrolled } \\
\text { women will receive two calls per week. }\end{array}$ & $\begin{array}{l}12 \text { weeks } \\
\text { between } \\
\text { midline and } \\
\text { endline } \\
\text { assessments }\end{array}$ & $\begin{array}{l}\text { Open-source } \\
\text { Interactive Voice } \\
\text { Response } \\
\text { Software (IVRS) }\end{array}$ \\
\hline
\end{tabular}


Table 2 RANI Intervention Timeline.

\begin{tabular}{|c|c|c|}
\hline No. & Activity & Timeline \\
\hline 1 & Intervention & \\
\hline 1.1 & T4 participatory education sessions & \\
\hline 1.1 .1 & $\begin{array}{l}\text { Monthly T4/Participatory learning action (PLA) sessions } \\
\& \text { Community Engagement Meetings }\end{array}$ & August $1^{\text {st }} 2019$ - July 2020 \\
\hline 1.2 & RANI Comms & \\
\hline 1.2 .1 & Monthly small group video screenings & August $1^{\text {st }} 2019-$ July 2020 \\
\hline 1.2 .2 & Monthly village-wide video screenings on large projectors & January 1,2020 - July 2020 \\
\hline 1.3 & Hemocue Testing and Demonstration & \\
\hline 1.3 .1 & $\begin{array}{l}\text { Monthly testing sessions \& demonstration of testing } \\
\text { results }\end{array}$ & August $1^{\text {st }} 2019$ - July 2020 \\
\hline 1.4 & Iron Folic Acid Monitoring & \\
\hline 1.4 .1 & Monthly supply point visit by community facilitator & August $1^{\text {st }} 2019$ - July 2020 \\
\hline 1.4 .2 & Quarterly demand and supply analysis and reporting & August $1^{\text {st }} 2019-$ July 2020 \\
\hline 1.4 .3 & IFA consumption monitoring & October 1st 2019 - July 2020 \\
\hline 1.4 .4 & On the spot IFA consumption & October 1st 2019 - July 2020 \\
\hline 1.5 & Government Interaction \& Dissemination of Findings & \\
\hline 1.5 .1 & $\begin{array}{l}\text { Monthly interaction with Block level Health Dept, } \\
\text { OLM/Panchayati Raj, Women and Child Development } \\
\text { Department }\end{array}$ & August $1^{\text {st }} 2019$ - July 2020 \\
\hline 1.5 .2 & $\begin{array}{l}\text { Monthly interaction with District Magistrate, Project } \\
\text { Director, Chief District Medical Officer and District } \\
\text { Social Welfare Officer }\end{array}$ & August $1^{\text {st }} 2019$ - July 2020 \\
\hline 1.5 .3 & $\begin{array}{l}\text { Monthly interaction with State Mission Director, Odisha } \\
\text { Livelihood Mission }\end{array}$ & August $1^{\text {st }} 2019-$ July 2020 \\
\hline
\end{tabular}

\section{Recruitment}

Within the selected clusters for data collection (described above), women between the ages of 15 and 49 residing in treatment $(n=2000)$ and control $(n=2000)$ clusters will be randomly selected and recruited to participate in the impact evaluation. Sampling will be stratified by treatment/control, village size and household.

To do so, we will create a household listing of eligible women within the selected clusters. The sampling size from each cluster will be proportional to population so that $60 \%$ of women in each arm come from highpopulation areas, 30\% come from medium population areas, and $10 \%$ come from low population areas. Once we determine the number of eligible women, we will sample every $\mathrm{n}^{\text {th }}$ household to get our total sample.

As mentioned and shown in Fig. 2, the sampling design also includes a greater-intensity subset of participants from which certain secondary outcomes will be measured (i.e., physical activity, work capacity, and socio-cognitive functioning). Procedures for this group are described below. Only non-pregnant women will participate in the greater-intensity activities for reasons related to participant burden. We will select the nonpregnant subset of women for these outcomes through the household listing based on proportion-to-size principles (and by considering costs to minimize travel by limiting the smallest sample size per village to at least 10 participants). Pregnant women will only be excluded from the three tests included as greater-intensity activities and anthropometric measurements, they are still eligible for hemoglobin measurements and the interview.

Everyone involved in the study (data collectors, the principal investigator, program implementers, project managers, etc.) except two staff members, will be blinded to who is in the treatment and control clusters. 


\section{Data Collection \& Measurement}

All participants $(n=2000$ in treatment and $n=2000$ in control clusters) will first undergo a point-of-care hemoglobin test to assess anemia status, followed by biometric assessments (height and weight), and a one-onone survey interview to assess demographic information, psychosocial factors, and anemia-related behaviors. In order to minimize interview time, a planned missingness design was used to create four shortened versions of the survey. All versions of the survey contain the main study outcomes, certain secondary outcomes, and basic demographics. Participants will be randomly assigned to receive one of the six versions. This process will significantly reduce participant burden (in comparison to having all participants answer all questions).

The following procedures will be occur for all participants inside their home.

\section{Hemoglobin measurements}

We will obtain hemoglobin levels from all participants, through point-of-care hemoglobin tests, using a HemoCue photometer (in line with India's National Family Health Survey methodology). This instrument provides hemoglobin levels immediately and accurately [24].

\section{Interview}

All participants will respond to a structured interview administered by a member of the local data collection team. This survey will measure self-reported IFA consumption and anemia status, as well as other secondary outcomes: knowledge, attitudes, and perceptions among participants; social norms; gender norms; mental health (via the CES-D scale); quality of life (via the SF-12); and diet (via the MDD-W questionnaire).

As mentioned, a smaller subset of non-pregnant women ( $n=150$ in each arm) will be randomly selected to provide further data on physical activity, work capacity, and socio-cognitive functioning. These measurements will be collected in a nearby community facility.

\section{Work capacity}

The Queens College Step Test assesses aerobic fitness [25]. The participant steps up and down on the 16.25-in. $(41.3 \mathrm{~cm})$ high platform at a rate of 22 steps per minute ( 88 beats per minute), assisted by the use of a metronome to maintain the right speed. Participants use a four-step cadence, 'up-up-down-down' for $3 \mathrm{~min}$, and heartbeat is assessed at five points: $t_{0}$ to $t_{4}$, corresponding to the beginning (before starting the step test), at the 1-, 2-, and 3-min marks, and then at the end (1 min after completing the step test). During our pilot study, we learned that the $16.25 \mathrm{in}$. height was too high for our sari-wearing participants and that a 12-in. height was found to be ideal. We will use this (12-in.) height in our study. Although using this lower height will not make our study readings directly comparable with other published studies, it will help us address our study objective (to compare longitudinally and across treatment-control arms).

\section{Socio-cognitive functioning}

We will also measure cognitive functioning within this sub-cohort through attention and working memory tasks. We will use the Simon Task and a Simple Reaction Time test to measure attention. We will also use a Corsi Blocks task and a Word Span test to assess working memory. To account for low computer literacy, these tests include both computer and non-computerized tests. Manual and computer based cognitive testing has successfully been carried out in several low and middle income countries in both rural and peri-urban settings including India [26-28]. All four tests will be administered by data collection staff who have been trained by the PI using a framework designed by an expert in cognitive testing.

\section{Physical activity}

Participants in the sub-cohort will be asked to wear an ActivPAL (PAL Technologies, LTD; Glasgow, UK) for three consecutive days to establish baseline measures of daily reclining, sitting, standing, and walking. The ActivPAL is small $(53 \times 35 \times 7 \mathrm{~mm})$, light-weight $(15 \mathrm{~g})$ and is attached to the mid-thigh.

\section{Statistical power and sample size calculations}

We assume a 7\% reduction in anemia (from 47 to $40 \%$ ), which is a lower effect size than typically found, alpha level of .05 with $80 \%$ power, the required sample size is 787 per arm $[29,30]$. Further assuming a design effect of 2.0 to account for clustering effects within villages, the required sample size with $20 \%$ loss to follow up is $N=$ 1968 per arm, which will be rounded up to 2000 per arm for a total of $N=4000$ across the treatment and control arms at baseline.

The more intense assessments consisted of three components - cognitive functioning, physical activity, and step test. We calculated the required sample size for the cognitive functioning component, assuming that cognitive functioning would improve by $16 \%$ and we assumed that ICC would not be an issue. This required a sample size of 288, which we rounded up to 300 . Sample size calculations for the other two components, ActivPal and Step Test, were not done separately because these tests are administered to the same sample as the cognitive test. The sample size matrix can be found in Table 3.

\section{Statistical analysis}

At baseline, we will conduct a series of bivariate tests across treatment and control arms. While randomization should ensure baseline matching, this is an effective 
Table 3 Sample Size Matrix

\begin{tabular}{lllllll}
\hline Study component & Intervention difference & Power & Alpha & Design effect & Required N & Rounded N \\
\hline Main trial & $7 \%$ anemia & $80 \%$ & 0.05 & 2 & 3936 & 4000 \\
Cognitive functioning & $16 \%$ more functioning & $80 \%$ & 0.05 & 1 & 288 & 300
\end{tabular}

technique to find any baseline difference. If any are found, they will be controlled for in any midline analysis. If we are unable to find baseline differences at midline, we will conduct a series of bivariate tests, including chisquare tests in which the binary outcome of anemia status at midterm is compared across treatment and control arms. This will be repeated at end line. Additionally, we will test the hypothesis that the treatment group will display a greater increase in hemoglobin count in comparison to the control group, supplemented with a difference-in-difference analysis in which change in hemoglobin levels between midline and baseline in the treatment group will be compared with corresponding changes in the control group. Thus, if there are differences across arms at baseline, we can still compare any observed change across arms. We will conduct another similar analysis at end line, using multivariate analysis of variance (MANOVA) techniques to uncover non-linear trends in the data. We will also evaluate the ability of the intervention to reduce anemia among pregnant versus non-pregnant women, as well as younger versus older women.

The primary analysis for hypothesis 1 will follow an intent-to-treat assumption. We will analyze data at the individual level, adjusting for clustering (at the village and cluster level), using generalized estimating equations (GEE). We will conduct a similar analysis through hierarchical linear modeling to account for village-level clustering effects, but this time with hemoglobin levels (continuous variable) as the dependent variable. In our MANOVA, time, treatment, and time $\mathrm{x}$ treatment will serve as the independent variables.

To test the hypothesis that social norms are the primary mediator between the intervention and change in hemoglobin levels, we will use structural equation models (SEM). The mediation analysis will include the secondary outcomes in the pathway between exposure to the intervention and hemoglobin level change.

Subsequent analyses will include other variables as outcomes, including those pertaining to physical activity; work capacity; quality of life; cognitive functioning; mental health; and psychosocial factors, including knowledge, attitude, normative beliefs, IFA intentions, and IFA use.

\section{Ethics and dissemination}

\section{Research ethics approval}

This study was approved by the George Washington University Institutional Review Board (IRB) and Sigma Science and Research, an IRB located in New Delhi,
India. The study was also reviewed and approved by Indian Council for Medical Research's (ICMR's) Health Ministry's Screening Committee (HMSC). Any changes to study protocol will be communicated with these regulatory entities for approval immediately.

\section{Participant consent and confidentiality}

Informed consent will be obtained in Oriya by local data collectors from DCOR Consulting. Data collectors will read the consent document to participants, who will then sign to indicate their consent. Participants under the age of 18 are required to obtain the written permission of one parent or legal guardian. All data from participants will be de-identified by the local principle investigator and stored in secure, password-protected computers that only the study team and its affiliates have access to.

\section{Dissemination}

In addition to disseminating our work at conferences and in peer-reviewed academic journals, we will disseminate findings through multi-media channels as peerreviewed findings get published. We will also update policy makers and stakeholders continuously with progress reports and newsletters. Finally, we will report findings back to the community where the research takes place after each data collection phase.

\section{Risk mitigation plan}

This is a low risk study so we do not anticipate serious adverse events. However, we will take precautions to ensure the safety of participants. Data collectors will be trained on HemoCue testing, how to communicate hemoglobin levels and anemia status to women following each test, and how to properly dispose of all HemoCue testing materials. While there will not be an independent assessment team to evaluate overall impact, we have put together an independent data safety and monitoring board (DSMB) to assess the outcomes on an ongoing process in order to ensure that the intervention does not inadvertently harm any participants.. The independent DSMB will review any serious adverse events and make recommendations for informing the IRB or stopping the study altogether. The DSMB includes researchers from India and the United States who will meet quarterly to review and discuss initial results from the study and any potential risks for participants. 


\section{Discussion}

While anemia has been a public health concern in India for decades, to our knowledge, no intervention has used a social-norms based model to encourage IFA use and iron rich food consumption. Our formative research findings showed the importance of shifting social norms among WRA and their primary referent groups (e.g., their husbands and mothers-in-law). We developed the T4 approach based on the formative research findings and the theory of normative social behavior. The longitudinal evaluation of the RANI T4 approach will both evaluate the efficacy of a norms-based intervention to increase uptake of IFA and iron-rich food as well as investigate the role of social norms as a mediator in anemiarelated behavior change.

We will encourage input from stakeholders throughout the implementation and evaluation of the RANI project. The promotion of IFA uptake is in line with the agenda of the Government of Odisha [16], the National Iron Plus Initiative of India [8], the WHO Sustainable Development Goals [7], and the Anemia Mukt Bharat [31]. The findings from this study can provide evidencebased methods to reduce anemia within the state of Odisha through an innovative social-norms lens. Findings may be applicable to other rural areas of India and South Asia.

The intervention design and implementation is based on tested theory and extensive formative research with the target community. A cluster-randomized controlled design improves internal validity. We minimized the risk of contamination between the intervention and control conditions by including a geographical buffer of one or two villages in between treatment and control clusters. As village clusters were randomly selected for inclusion into the study, and then randomly assigned into treatment and control arms, both between- and within- cluster variation should be similar across both treatment and control groups. The inclusion of a control group further improves the internal validity of this study as it allows for the consideration of secular trends, which is particularly important in Odisha, India where ongoing efforts to reduce anemia by other groups can introduce history bias.

Additionally, a longitudinal evaluation design provides a better understanding of hemoglobin level changes with IFA use. The use of three time points also allows for the investigation of non-linear trends in hemoglobin levels after exposure to the intervention.

Blinding at two levels may strengthen the quality of our data. At the first level, the intervention implementers have been blinded to which villages are selected for data collection. This will minimize bias in implementation, allowing for our selected sample for data collection to be representative of all the villages that received the RANI treatment. At the second level, the data collectors will be unaware of which villages are selected for treatment and control. This will also minimize any potential recording bias as the data collectors will not know if they are currently collecting data from an individual in the treatment or control arm.

\section{Limitations}

To our knowledge, this is the first study that tests the effects of a norms-based intervention to improve IFA demand among WRA in India through a randomized controlled trial. As a result, we use a "kitchen sink" approach, in which we assess the overall impact of all intervention components, without distinguishing which component may have been most effective. However, we will measure exposure to components of the intervention as well as collect monitoring and processes evaluation data to understand the effects of specific intervention activities to the extent possible.

Additionally, this is primarily a demand-side intervention. Our formative research shows that access to IFA is not a major barrier to use, but if our intervention is successful, an increase in demand may impact supply. If supply chain problem occurs, changes in social norms may have limited impact. Therefore, we will be monitoring stock-outs throughout the intervention and we will use monitoring and evaluation data at the block level to advocate for additional supply if/when demand increases. The study length supports an evaluation of the short-term effectiveness of the intervention but does not evaluate longer-term sustainability of the social norms or behavior changes.

The current Indian guidelines suggest IFA supplementation once a week for non-pregnant women and once a day for pregnant women in their second and third trimester (both comprised of $60 \mathrm{mg}$ elemental Iron +500 mcg Folic Acid) [16]. Abdominal pain is commonly reported among women who take IFA daily, but less frequently in women who take IFA weekly [32]. As pregnant women experience the side effects associated with daily dosage, the reputation of IFA may dampen and reduce demand. Previous studies show that women who experience side effects, such as abdominal pain, or believe that they are caused by IFA are less likely to adhere to IFA than those who do not $[33,34]$. Dosage regimen also influences absorption of iron; clinical studies have shown that IFA with $\geq 60 \mathrm{mg}$ iron administered daily increases hepcidin, subsequently reducing absorption on the next day [35]. However, IFA administered on alternating days yielded approximately twice the amount of iron absorption than daily administration [36].

It is important to note that we only include women in this study's impact evaluation - understanding normative change among men/husbands would add valuable information to this study. Additionally, the intervention 
does not target the behaviors of frontline workers; therefore frontline workers may continue practices of distributing IFA only to pregnant women or failing to follow up on IFA adherence.

Additionally, we anticipate that attrition may occur through the course of this study. To minimize any effects to internal validity, the power size calculations were conducted with $20 \%$ anticipated attrition. If attrition does occur, we will investigate if systematic differences are observed in baseline among those who drop out of the study.

\section{Abbreviations}

IFA: Iron-folic acid; RCT: Randomized controlled trial; WHO: World health organization; WRA: Women of reproductive health; NIPI: National iron plus initiative; RANI: Reduction in anemia through normative innovations: SHG: Self-help groups; TNSB: Theory of normative social behavior

\section{Acknowledgments}

Not applicable.

\section{Authors' contributions}

HY made contributions to the sampling and recruitment procedures, as well as the data collection procedures. HY also played a large role in drafting and revising the protocol. ES made contributions to the conception and all parts of the design, as well as played a large role in drafting the protocol. RR was responsible for the conception of the study; the development of the research questions, objectives, and rational; and the design of the study. $\mathrm{He}$ also played a large role in revising the protocol. IP, AM, and SM all played a role in drafting components of the protocol and revising it. All authors have read and approved the manuscript.

\section{Funding}

This work was supported by a grant from The Bill and Melinda Gates Foundation (OPP1182519) to the George Washington University, Rajiv N. Rimal, principal investigator. The funders had no role in study design, data collection and analysis, decision to publish, or preparation of the manuscript.

\section{Availability of data and materials}

Data sharing is not applicable to this article as no datasets were generated or analyzed during the current study. The dataset(s) that will come out of this study will be available in the Gates Open Access repository.

\section{Ethics approval and consent to participate}

As mentioned, study was approved by the George Washington University Institutional Review Board (IRB) and Sigma Science and Research, an IRB located in New Delhi, India. The study has also been reviewed and approved by the Indian Council for Medical Research's (ICMR's) Health Ministry's Screening Committee (HMSC). Any changes to study protocol will be communicated with these regulatory entities for approval immediately. Participants will be required to provide informed consent, or parental permission if under 18 years old; consent procedures have been approved by the IRBs.

\section{Consent for publication}

Not applicable.

\section{Competing interests}

The authors declare that they have no competing interests.

\section{Author details}

${ }^{1}$ Department of Prevention and Community Health, George Washington University Milken Institute School of Public Health, Washington, District of Columbia, USA. ${ }^{2}$ Department of Health, Behavior and Society, Johns Hopkins University Bloomberg School of Public Health, Baltimore, MD, USA. ${ }^{3}$ Department of Social and Economic Empowerment, IPE Global Limited, New Delhi, Delhi, India. ${ }^{4}$ DCOR Consulting, Bhubaneswar, India.
Received: 10 July 2019 Accepted: 24 January 2020

Published online: 07 February 2020

\section{References}

1. International Institute for Population Sciences (IIPS) and ICF. National Family Health Survey (NFHS-4), India, 2015-16: Odisha. Mumbai: IIPS; 2017.

2. Horton S, Ross J. The economics of iron deficiency. Food Policy. 2003;28(1): $51-75$.

3. Scholl TO, Hediger ML, Fischer RL, Shearer JW. Anemia vs iron deficiency: increased risk of preterm delivery in a prospective study. Am J Clin Nutr. 1992:55(5):985-8.

4. Casey GJ, Phuc TQ, MacGregor L, Montresor A, Mihrshahi S, Thach TD, Tien NT, Biggs BA. A free weekly iron-folic acid supplementation and regular deworming program is associated with improved hemoglobin and iron status indicators in Vietnamese women. BMC Public Health. 2009;9(1):261.

5. Preziosi P, Prual A, Galan P, Daouda H, Boureima H, Hercberg S. Effect of iron supplementation on the iron status of pregnant women: consequences for newborns. Am J Clin Nutr. 1997:66(5):1178-82.

6. Wiegersma AM, Dalman C, Lee BK, Karlsson H, Gardner RM. Association of prenatal maternal anemia with neurodevelopmental disorders. JAMA Psychiatry. 2019;76(12):1294-304.

7. World Health Organization. Global nutrition targets 2025: anaemia policy brief. Geneva: Department of Nutrition for Health and Development; 2014

8. Kapil U, Bhadoria AS. National Iron-plus Initiative guidelines for control of iron deficiency anaemia in India, 2013. Natl Med J India. 2014;27(1):27-9.

9. Ministry of Health and Family Welfare. Guidelines for control of Iron deficiency Anaemia: National Iron+ initiative. New Delhi: Government of India Adolescent Division; 2013.

10. Shet AS, Zwarenstein M, Rao A, Jebaraj P, Arumugam $K$, Atkins S, et al. Effect of a community health worker-delivered parental education and counseling intervention on Anemia cure rates in rural Indian children: a pragmatic cluster randomized clinical trial. JAMA Pediatr. 2019;173(9):826-34.

11. Singh $M$, Honnakamble RA, Rajoura OP. Knowledge, attitude and practice change about Anemia after intensive health education among adolescent school girls of Delhi: an intervention study. Int J Med Public Health. 2019; 9(3):71-3.

12. World Health Organization. Weekly iron and folic acid supplementation programmes for women of reproductive age: an analysis of best programme practices. Geneva: WHO Western Pacific Regional; 2011.

13. Hazra A, Atmavilas Y, Hay K, Saggurti N, Verma RK, Ahmad J, ... Irani L. Effects of health behaviour change intervention through women's self-help groups on maternal and newborn health practices and related inequalities in rural India: a quasi-experimental study. EClinicalMedicine. 2019;18:100198.

14. Government of India. Census of India: 2011. New Delhi: Office of the Registrar General \& Census Commissioner; 2012.

15. Sedlander E, Rimal RN, Talegawkar SA, Yilma H, Munar W. The RANI project: a socio-normative intervention to reduce anemia in Odisha. India: A formative research protocol. Gates Open Research; 2018. p. 2.

16. Intensified National Iron Plus Initiative (I-NIPI). Ministry of Health and Family Welfare. Anemia Mukt Bharat: Government of India; 2018.

17. Cialdini RB, Kallgren CA, Reno RR. A focus theory of normative conduct: a theoretical refinement and reevaluation of the role of norms in human behavior. Adv Exp Soc Psychol. 1991:201-34.

18. Rimal RN, Lapinski MK. A re-explication of social norms, ten years later. Communication Theory. 2015;25(4):393-409.

19. Rimal RN, Real K. How behaviors are influenced by perceived norms: a test of the theory of normative social behavior. Commun Res. 2005;32(3):389414.

20. Rimal RN. Modeling the relationship between descriptive norms and behaviors: a test and extension of the theory of normative social behavior (TNSB). Health Commun. 2008:23(2):103-16.

21. Carcioppolo N, Orrego Dunleavy V, Yang Q. How do perceived descriptive norms influence indoor tanning intentions? An application of the theory of normative social behavior. Health Commun. 2017;32(2):230-9.

22. Walker DD, Neighbors C, Rodriguez LM, Stephens RS, Roffman RA. Socia norms and self-efficacy among heavy using adolescent marijuana smokers. Psychol Addict Behav. 2011:25(4):727.

23. Woolf J, Rimal RN, Sripad P. Understanding the influence of proximal networks on high school athletes' intentions to use androgenic anabolic steroids. J Sport Manag. 2014;28(1):8-20. 
24. Sanchis-Gomar F, Cortell-Ballester J, Pareja-Galeano H, Banfi G, Lippi G. Hemoglobin point-of-care testing: the HemoCue system. J Lab Automation. 2012;18(3):198-205.

25. Mcardle WD, Katch FI, Pechar GS, Jacobson LONI, Ruck S. Reliability and interrelationships between maximal oxygen intake, physical work capacity and step-test scores in college women. Med Sci Sports. 1972:4(4):182-6.

26. Murray-Kolb LE, Wenger MJ, Scott SP, Rhoten SE, Lung'aho MG, Haas JD. Consumption of Iron-biofortified beans positively affects cognitive performance in 18- to 27-year-old Rwandan female college students in an 18-week randomized controlled efficacy trial. J Nutr. 2017;jn255356. https:// doi.org/10.3945/jn.117.255356.

27. Gupta K, Savita, Agnihotri S, Telles S, Balkrishna A. Performance in a Corsi block-tapping task following high-frequency yoga breathing or breath awareness. Int J Yoga. 2019;12(3):247-51.

28. Scott SP, Murray-Kolb LE, Wenger MJ, Udipi SA, Ghugre PS, Boy E, Haas JD. Cognitive performance in Indian school-going adolescents is positively affected by consumption of Iron-biofortified pearl millet: a 6-month randomized controlled efficacy trial. J Nutr. 2018;148(9):1462-71. https://doi. org/10.1093/jn/nxy113.

29. Bharti S, Bharti B, Naseem S, Attri SV. A community-based cluster randomized controlled trial of "directly observed home-based daily iron therapy" in lowering prevalence of anemia in rural women and adolescent girls. Asian Pac J Public Health. 2015;27:1333-44.

30. Rivera JA, Sotres-Alvarez D, Habicht JP, Shamah T, Villalpando S. Impact of the Mexican program for education, health, and nutrition (Progresa) on rates of growth and anemia in infants and young children: a randomized effectiveness study. Jama. 2004;291(21):2563-70.

31. Government of Odisha. Odisha multi-Sectoral nutrition action plan (ONAP) 2017-2020. India: Odisha; 2016

32. Joshi M, Gumashta R. Weekly iron folate supplementation in adolescent girls-an effective nutritional measure for the management of iron deficiency anaemia. Global J Health Sci. 2013;5(3):188.

33. Bhatt RJ, Mehta HK, Khatri V, Chhaya J, Rahul K, Patel P. A study of access and compliance of iron and folic acid tablets for prevention and cure of anaemia among adolescent age group females in Ahmedabad district of India surveyed under multi indicator cluster survey. Glob J Med Public Health. 2011;2(4):1-6.

34. Ratanasiri T, Koju R. Effect of knowledge and perception on adherence to iron and folate supplementation during pregnancy in Kathmandu, Nepal. J Med Assoc Thail. 2014;97(10):S67-74.

35. Moretti D, Goede JS, Zeder C, Jiskra M, Chatzinakou V, Tjalsma H, et al. Oral iron supplements increase hepcidin and decrease iron absorption from daily or twice-daily doses in iron-depleted young women. Blood, J Am Soc Hematol. 2015;126(17):1981-9.

36. Stoffel NU, Zeder C, Brittenham GM, Moretti D, Zimmermann MB. Iron absorption from supplements is greater with alternate day than with consecutive day dosing in iron-deficient anemic women. Haematol, haematol-2019. 2019.

\section{Publisher's Note}

Springer Nature remains neutral with regard to jurisdictional claims in published maps and institutional affiliations.

Ready to submit your research? Choose BMC and benefit from:
- fast, convenient online submission
- thorough peer review by experienced researchers in your field
- rapid publication on acceptance
- support for research data, including large and complex data types
- gold Open Access which fosters wider collaboration and increased citations
- maximum visibility for your research: over 100M website views per year
At BMC, research is always in progress.
Learn more biomedcentral.com/submissions

